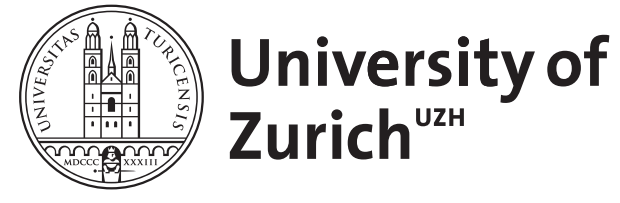

\title{
Are star formation rates of galaxies bimodal?
}

Feldmann, Robert

\begin{abstract}
Star formation rate (SFR) distributions of galaxies are often assumed to be bimodal with modes corresponding to star-forming and quiescent galaxies, respectively. Both classes of galaxies are typically studied separately, and SFR distributions of star-forming galaxies are commonly modelled as lognormals. Using both observational data and results from numerical simulations, I argue that this division into star-forming and quiescent galaxies is unnecessary from a theoretical point of view and that the SFR distributions of the whole population can be well fitted by zero-inflated negative binomial distributions. This family of distributions has three parameters that determine the average SFR of the galaxies in the sample, the scatter relative to the star-forming sequence and the fraction of galaxies with zero SFRs, respectively. The proposed distributions naturally account for (i) the discrete nature of star formation, (ii) the presence of 'dead' galaxies with zero SFRs and (iii) asymmetric scatter. Excluding 'dead' galaxies, the distribution of $\log$ SFR is unimodal with a peak at the star-forming sequence and an extended tail towards low SFRs. However, uncertainties and biases in the SFR measurements can create the appearance of a bimodal distribution.
\end{abstract}

DOI: https://doi.org/10.1093/mnrasl/slx073

Posted at the Zurich Open Repository and Archive, University of Zurich ZORA URL: https://doi.org/10.5167/uzh-143828

Journal Article

Published Version

Originally published at:

Feldmann, Robert (2017). Are star formation rates of galaxies bimodal? Monthly Notices of the Royal Astronomical Society. Letters, 470(1):59-63.

DOI: https://doi.org/10.1093/mnrasl/slx073 


\title{
Are star formation rates of galaxies bimodal?
}

\author{
Robert Feldmann ${ }^{\star}$ \\ Institute for Computational Science, University of Zurich, CH-8057 Zurich, Switzerland
}

Accepted 2017 May 8. Received 2017 May 8; in original form 2017 April 11

\begin{abstract}
Star formation rate (SFR) distributions of galaxies are often assumed to be bimodal with modes corresponding to star-forming and quiescent galaxies, respectively. Both classes of galaxies are typically studied separately, and SFR distributions of star-forming galaxies are commonly modelled as lognormals. Using both observational data and results from numerical simulations, I argue that this division into star-forming and quiescent galaxies is unnecessary from a theoretical point of view and that the SFR distributions of the whole population can be well fitted by zero-inflated negative binomial distributions. This family of distributions has three parameters that determine the average SFR of the galaxies in the sample, the scatter relative to the star-forming sequence and the fraction of galaxies with zero SFRs, respectively. The proposed distributions naturally account for (i) the discrete nature of star formation, (ii) the presence of 'dead' galaxies with zero SFRs and (iii) asymmetric scatter. Excluding 'dead' galaxies, the distribution of log SFR is unimodal with a peak at the star-forming sequence and an extended tail towards low SFRs. However, uncertainties and biases in the SFR measurements can create the appearance of a bimodal distribution.
\end{abstract}

Key words: galaxies: evolution - galaxies: formation -galaxies: star formation.

\section{INTRODUCTION}

The bimodal colour distribution of nearby galaxies leads to a natural classification into 'blue' and 'red' galaxies (e.g. Baldry et al. 2004; Brammer et al. 2009). On average, blue (red) galaxies in the local Universe have high (low) star formation rates (SFRs), although dust extinction complicates this basic picture (Whitaker et al. 2012; Taylor et al. 2014). Hence, galaxies are often divided into starforming and quiescent galaxies based on their level of star formation activity (e.g. Balogh et al. 2004; Moustakas et al. 2013). However, whether the distribution of SFRs is also bimodal remains an open question (e.g. Elbaz et al. 2007; McGee et al. 2011).

The SFRs of star-forming galaxies strongly correlate with their stellar masses, resulting in a well-defined 'star-forming sequence' (Noeske et al. 2007). In contrast, quiescent galaxies have generally very low (or vanishing levels) of SFRs whose exact amount is challenging to infer observationally (e.g. Brinchmann et al. 2004; Utomo et al. 2014; Chang et al. 2015). Hence, most observational studies focus on the SFRs of 'star-forming galaxies' alone and model their distribution at fixed stellar mass with a lognormal distribution (e.g. Noeske et al. 2007) or with the sum of two lognormal distributions (Sargent et al. 2012). The intrinsic scatter around the star-forming sequence is found to be about $\sim 0.3-0.4$ dex, essentially independent of redshift $(z \sim 0-6$; e.g. Chang et al. 2015; Salmon et al. 2015; Schreiber et al. 2015; Shivaei et al. 2015). The approach of approximating the distribution of SFRs of star-forming galaxies with a lognormal is also adopted in most analyses of galaxy forma-

*E-mail: feldmann@physik.uzh.ch tion simulations (e.g. Schaye et al. 2015; Sparre et al. 2015; Dave, Thompson \& Hopkins 2016; Feldmann et al. 2016).

However, this approach has a number of shortcomings. First, a clear separation of galaxies into star forming and quiescent is challenging in practice. Classifications based on colour-magnitude diagrams suffer from a large population of dust-obscured star-forming galaxies with colours intermediate between blue and red (Salim et al. 2009; Taylor et al. 2014; Chang et al. 2015). While colourcolour diagrams offer a more robust alternative (Wuyts et al. 2007), the mapping from colours to SFRs can be biased by relatively small amounts of recent star formation, by dust and by the presence of evolved stellar populations (e.g. Salim et al. 2009; Wuyts et al. 2011; Fumagalli et al. 2014). For instance, while high-redshift galaxies classified as quiescent based on colour-colour diagrams have typically significantly reduced SFRs (Man et al. 2016; Straatman et al. 2016), some of them show non-negligible levels of SFR and dust extinction (e.g. Brammer et al. 2009; Spitler et al. 2014; Mancini et al. 2015). It is thus legitimate to ask whether quiescent and starforming galaxies are actually two separate populations or whether galaxies simply form a continuum from low to high specific SFRs (sSFRs) without a natural dividing point.

Secondly, a lognormal distribution predicts a symmetric scatter around the star-forming sequence, in contrast to the findings of many observational studies and numerical simulations (Brinchmann et al. 2004; Chang et al. 2015; Dave et al. 2016). Often, this difference is attributed to an 'imperfect separation' into star-forming and quiescent galaxies (e.g. Chang et al. 2015). Instead, I argue that the level of asymmetry of the scatter contains important information about galactic star formation and should not be ignored. 
Thirdly, star formation is correlated in space and time as stars are typically born in clusters (Lada \& Lada 2003). These correlations introduce a level of discreteness into the star formation process. For instance, star clusters in the Milky Way have masses ranging from $\sim 10^{2}$ to $10^{3} \mathrm{M}_{\odot}$ for open clusters to $\sim 10^{5} \mathrm{M}_{\odot}$ for young massive clusters (Portegies Zwart, McMillan \& Gieles 2010). Star clusters can be even more massive in star-bursting galaxies (Zhang \& Fall 1999) and at high redshift, when galaxies are generally more gas rich (e.g. Swinbank et al. 2010; Kruijssen 2012). The discreteness effect is further exacerbated in observationally inferred SFRs as those trace energy injections from rare, massive stars (Kennicutt 1998). A lognormal distribution, which is a continuous distribution, does not account for this discrete mode of star formation.

Finally, the star formation activity, especially for higher redshift and/or lower mass galaxies, is thought to be bursty (e.g. Dominguez et al. 2015; Sparre et al. 2017). Consequently, many galaxies may experience intermittent episodes of low or vanishing SFR (Feldmann et al. 2017). However, galaxies with fully suppressed SFRs cannot be modeled by a lognormal distribution. Thus, in practice, galaxies with SFRs below the detection limit are excluded from (non-stacked) analyses of the star-forming sequence even if such galaxies are star forming according to their colours (e.g. Whitaker et al. 2014).

As I argue in this Letter, these shortcomings can be mitigated by dropping the assumption of a lognormal SFR distribution and by not dividing galaxies into star-forming and quiescent galaxies in the first place. In particular, I propose to replace the lognormal ansatz with (zero-inflated) negative binomial distributions (NBDs). This family of distributions found wide applicability in particle physics (e.g. multiplicity distributions of charged particles in hadronic collisions; Alner et al. 1985), astrophysics (e.g. the number of globular clusters in galaxies and event rates of fast radio bursts; De Souza et al. 2015; Wiel et al. 2016) and cosmology (e.g. modelling count-in-cell distributions and void probability functions; Carruthers \& DuongVan 1983; Gaztanaga 1992) but, to my knowledge, has not been used to model distributions of SFRs or sSFRs.

Choosing an appropriate model for the distributions of SFRs is not self-evident as the origin of the scatter around the star-forming sequence is not well understood. As gas accretion on to galaxies and star formation within galaxies are likely linked (e.g. Bouché et al. 2010; Sánchez Almeida et al. 2014), the scatter may be related to variations in the gas accretion rates (Dutton, van den Bosch \& Dekel 2010; Forbes et al. 2014) or halo growth rates (Feldmann \& Mayer 2015; Feldmann et al. 2016; Rodríguez-Puebla et al. 2016). The scatter may also arise from random stochasticity (Kelson 2014), gas fraction variations (e.g. Magdis et al. 2012; Saintonge et al. 2012; Scoville et al. 2016), changes in the efficiency of star formation (e.g. Genzel et al. 2010; Saintonge et al. 2012), a natural diversity in star formation histories (Gladders et al. 2013; Dressler et al. 2016) or combinations of some of these processes (e.g. Tacchella et al. 2016; Feldmann et al. 2017). Hence, it appears justified to explore empirically how well SFRs follow various basic distributions.

With the recent availability of large numbers of reliable SFR measurements, these basic distributions can be compared against observations and numerical simulations. I use spectral energy distribution (SED) based SFR estimates of galaxies in the local Universe from the Sloan Digital Sky Survey (SDSS) (Chang et al. 2015), ultraviolet (UV) and infrared (IR) based SFR measurements of galaxies at $z \sim 2$ from 3D-HST (Brammer et al. 2012; Skelton et al. 2014), as well as SFRs measured in cosmological simulations (Feldmann et al. 2016) that are part of the Feedback in Realistic Environments (FIRE) Project (Hopkins et al. 2014).
This Letter is organized as follows: Section 2 introduces probability distributions to model SFRs in galaxy samples. Section 3 shows that zero-inflated NBDs (zNBDs) provide adequate approximations to SFR distributions in observations and numerical simulations. I discuss the implications for a possible SFR bimodality in the final section.

\section{MODELLING THE DISTRIBUTION OF SFRS}

At fixed stellar mass, SFRs of star-forming galaxies are typically assumed to obey lognormal distributions (e.g. Chang et al. 2015), i.e. the $\operatorname{logarithm}^{1}$ of the SFR is assumed to be a continuous variable that is normally distributed with standard deviation $\sigma \equiv \sigma_{\ln S F R}=$ $\sigma_{\lg S F R} \ln 10$. The mean, median and most probable value of $\lg$ SFR coincide, and they define the position of the star-forming sequence for the given stellar mass.

Instead, I propose to model SFRs of galaxies with NBDs. As NBDs describe count data, I assume that the star formation activity over time $t_{\mathrm{av}}$ consists of individual star formation events, each adding mass $m_{\mathrm{SFC}}$, i.e.

$\mathrm{SFR}=\mathrm{SFC} m_{\mathrm{SFC}} / t_{\mathrm{av}}$.

SFC ('star formation count') is a non-negative integer-valued random variable with a [potentially zero-inflated (discussed later)] NBD.

The probability mass function (PMF) of an NBD is specified by two parameters, for example, the expected count $\mu$ and a shape parameter $\theta$, both positive real numbers. The probability of the outcome $\mathrm{SFC}=k \in \mathbb{N}$ is (e.g. Cameron \& Trivedi 2013)

$P_{\mathrm{NB}}(k ; \mu, \theta)=\left(\begin{array}{c}\theta+k-1 \\ \theta-1\end{array}\right)\left(\frac{\mu}{\theta+\mu}\right)^{k}\left(\frac{\theta}{\theta+\mu}\right)^{\theta}$.

If $\theta$ is a positive integer, equation (2) describes the probability distribution of the number of Bernoulli trials (each with a success probability $\theta /[\theta+\mu]$ ) undertaken before the $\theta$ th trial is successful. Cases with $\theta=1$ are known as geometric distributions. There exist many other characterizations of NBDs (Boswell \& Patil 1970). For the remainder of this Letter, I will assume $\theta=O(1)$ and $\mu \gg \theta$.

NBDs are often used to model count data that is 'overdispersed' relative to a Poisson distribution. ${ }^{2}$ NBDs are Poisson-Gamma mixtures, which suggests a simple physical interpretation: The overdispersion arises from galaxy-by-galaxy variations of the expected number of SFCs even among galaxies of the same stellar mass. This is plausible as the expected number of SFCs in a galaxy should vary depending on the state of its interstellar medium.

Zero-inflated models (Mullahy 1986; Lambert 1992) increase the probability of obtaining zero counts, i.e. of producing galaxies with zero SFRs. For zNBDs,

$P_{\mathrm{zNB}}(k ; \pi, \mu, \theta)=\pi \delta_{0 k}+(1-\pi) P_{\mathrm{NB}}(k ; \mu, \theta)$,

where $\pi \in[0,1]$ parametrizes the excess probability to obtain zero counts: $P_{\mathrm{zNB}}(0 ; \pi, \mu, \theta)-P_{\mathrm{NB}}(0 ; \mu, \theta)=\pi\left[1-P_{\mathrm{NB}}(0 ; \mu, \theta)\right]$. Candidate processes responsible for $\pi>0$ include galactic outflows powered by starbursts or active galactic nuclei (e.g. King \& Pounds

\footnotetext{
${ }^{1}$ In the following, $\log , \lg$ and $\ln$ denote the logarithm to an arbitrary base, to base 10 and to base e, respectively.

${ }^{2}$ A Poisson-distributed random variable has a variance equal to its mean value $\mu$. In contrast, the variance of a random variable with an NBD is equal to $\mu+\mu^{2} / \theta$, i.e. the variance at given $\mu$ can be adjusted by changing $\theta$.
} 

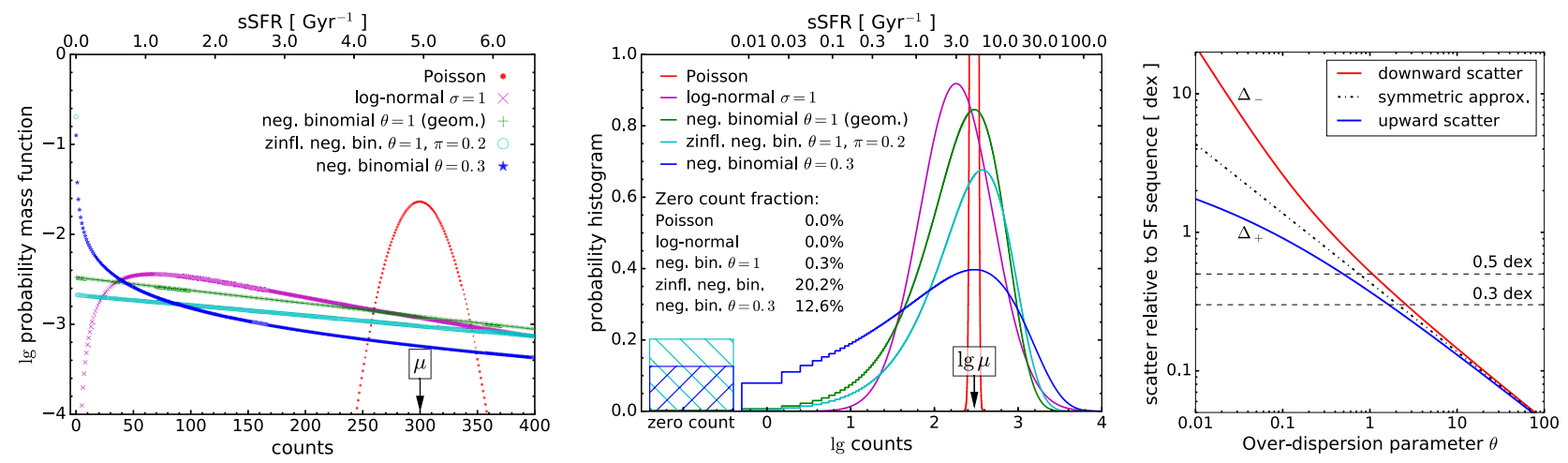

Figure 1. Properties of negative binomial, zero-inflated negative binomial, lognormal and Poisson distributions. All distributions have the same mean number of counts $(\mu=300)$. For illustration purposes, I convert between counts (bottom axis) and sSFR (top axis) by assuming that each count corresponds to a fractional increase of galactic stellar mass by $3.3 \times 10^{-4}$ over the past 20 Myr. Left-hand panel: PMFs of all considered distributions are unimodal. The PMF of an NBD with $\theta \leq 1$ decreases monotonically with increasing counts. For large $\mu$, the PMF of a Poisson distribution approximates a normal distribution with mean $=$ variance $=\mu$. Middle panel: probability density function (PDF) of log counts. All distributions show a well-defined 'star-forming sequence' with a peak close to $\lg \mu$. Compared with the observed star-forming sequence, a Poisson distribution predicts a much narrower star-forming sequence. NBDs with $\theta=1$ predict a reasonable amount of scatter and a negligible fraction of galaxies with zero SFRs. NBDs with $\theta<1$ result in a strongly broadened star-forming sequence and in a significant number of non-star-forming galaxies. Zero-inflated variants of the shown distributions boost the probability of having zero counts. Right-hand panel: upward $\left(\Delta_{+}\right)$and downward $\left(\Delta_{-}\right)$scatter relative to the peak of the lg count distribution as a function of $\theta$ for NBDs (see the text). The downward scatter is generally larger than the upward scatter. The dot-dashed curve shows the approximation $\theta^{-1 / 2} / \ln 10$, which holds if $1 \lesssim \theta \ll \mu$. NBDs with $\theta \sim 0.3-3$ have a scatter relative to the peak of the star-forming sequence of about $0.2-1$ dex.

2015; Somerville \& Davé 2015, and references therein) and strong environmental effects (Gunn \& Gott 1972).

Examples of lognormal, negative binomial, zero-inflated negative binomial and Poisson distributions are shown in Fig. 1. For illustrative purposes, I convert between PMFs and probability density distributions (PDFs) by approximating probability point masses with intervals of uniform probability density. As the figure shows, a count of zero is the most probable outcome for random variables with (z)NBDs if $\theta \leq 1$. Interestingly, while the probability of obtaining a certain count value is unimodal and decreases monotonically with the count value for (z)NBDs, the distribution of the logarithm of the count variable shows a well-defined peak near ${ }^{3}$ the logarithm of $\mu$. In addition, a conversion to $\log$ counts requires that zero and non-zero counts are treated as separate components. As I argue in Section 4, this split into two components may lead to an apparent 'bimodality' of log SFR distributions.

Fig. 1 also illustrates that the distribution of log counts around the peak is asymmetric for (z)NBDs. There is a significant tail towards lower values, resulting in a non-negligible probability of a zero count outcome if $\theta<1$. Furthermore, the degree of asymmetry and the width of the log count distribution increases with decreasing $\theta$ (see the right-hand panel). There, I plot how the upward scatter $\left(\Delta_{+}\right)$and the downward $\left(\Delta_{-}\right)$scatter ${ }^{4}$ relative to the peak of the PDF scale with $\theta$. For $\theta<1$, upward scatter and downward scatter differ significantly from each other, while for $\theta \gtrsim 1, \Delta_{+} \sim \Delta_{-} \sim \theta^{-1 / 2} / \ln 10$.

\footnotetext{
${ }^{3}$ Provided $\mu \gg \theta$, the peak of $\lg$ count is near $\lg (\mu+\theta / 2) \sim \lg \mu$. In contrast, for a lognormally distributed random variable $X$, the distributions of $\lg X$ and $X$ peak at $\lg \mu-\frac{\sigma^{2}}{2 \ln 10}$ and $\mu / \mathrm{e}^{1.5 \sigma^{2}}$, respectively.

${ }^{4} \Delta_{+}$and $\Delta_{-}$are defined as follows. Let $\lg c_{*}$ denote the position where the PDF of $\lg$ counts reaches its maximum value $p_{*}$. Increasing (decreasing) the counts by $\Delta_{+}\left(\Delta_{-}\right)$dex relative to $c_{*}$ results in a decrease of the PDF by $\chi=\mathrm{e}^{-1 / 2}$ relative to $p_{*}$. The factor $\mathrm{e}^{-1 / 2}$ is chosen such that $\Delta_{+}=\Delta_{-}=1$ standard deviation for a normal distributed PDF of lg counts. For $\mu \gg \theta$, $\Delta_{ \pm}=\lg \left(-W_{\mp}\left(-\chi^{1 / \theta} / \mathrm{e}\right)\right)$, where $W_{+}$and $W_{-}$are the principal and the -1 branch of the Lambert $W$ function, respectively.
}

\section{COMPARISON WITH OBSERVATIONS AND SIMULATIONS}

I use three samples of galaxies at different redshifts, with different stellar masses and from different sources to test whether SFR distributions at fixed stellar mass can be approximated by zNBDs.

The first sample comprises 38246 nearby galaxies from SDSS. SFR and stellar mass estimates are based on multiwavelength (UV to mid-IR) SED modelling (Chang et al. 2015). I select a masscomplete sample of galaxies ${ }^{5}$ with stellar masses in the range of $10^{10}-10^{11} \mathrm{M}_{\odot}$ and with redshifts $z<0.05456$. I set the SFR of a galaxy to zero if the best-fitting sSFR is very low $\left(<3 \times 10^{-12} \mathrm{yr}^{-1}\right)$ and the modelling error is at least twice the spread of the starforming sequence.

The second sample contains 2317 galaxies at $z \sim 2$ from the 3DHST survey (Brammer et al. 2012; Skelton et al. 2014; catalogue v4.1). SFRs in the catalogue are based on $U V+24 \mu \mathrm{m}$ luminosities (SFRs of non-detected galaxies are set to zero), while stellar masses are derived from SEDs fits. I select all galaxies ${ }^{6}$ from the five available fields with stellar masses in the range of $10^{10}-10^{11} \mathrm{M}_{\odot}$ and with redshifts $z=1.5-2$. For both observational samples, I convert SFR into SFC with the help of equation (1) and by adopting a conversion factor ${ }^{7}$ of $m_{\mathrm{SFC}} / t_{\mathrm{av}}=10^{-3} \mathrm{M}_{\odot} \mathrm{yr}^{-1}$.

The last sample combines SFRs and stellar masses from cosmological galaxy simulations. Specifically, I use $1648 z=6$ galaxies with $M_{\text {star }}=10^{7}-10^{9} \mathrm{M}_{\odot}$ from the MassiveFIRE simulation suite (Feldmann et al. 2016, 2017). Gas and stellar components of the simulated galaxies are represented by gas and star particles with

\footnotetext{
${ }^{5}$ Excluding galaxies with flag $\neq 1$, i.e. those without reliable aperture corrections, WISE photometry or SED fits.

${ }^{6}$ Excluding galaxies with star_flag $=1$, near_star $=1$ or use_phot $\neq 1$.

${ }^{7}$ This choice corresponds to, for example, $m_{\mathrm{SFC}}=2 \times 10^{4} M_{\odot}$ and $t_{\mathrm{av}}=20$ Myr. However, provided $k \gg 1$ and $\mu \gg \theta$, (z)NBDs are well approximated by (zero-inflated) gamma distributions, and the PDF of $\lg$ SFR for SFR $>0$ approaches $\ln (10) x P_{\text {Gamma }}(x ; \alpha=\theta, \beta=1)$ with $x=\theta \mathrm{SFR} /\langle\mathrm{SFR}\rangle$, i.e. it is independent of the conversion factor.
} 
masses $m_{\mathrm{b}}=3.3 \times 10^{4} \mathrm{M}_{\odot}$. Star formation occurs probabilistically based on the local conditions of the interstellar medium. Each individual star formation event results in the formation of a star particle of mass $m_{\mathrm{b}}$. I refer the reader to Hopkins et al. (2014) for a background on the simulation methodology. Stellar masses and SFRs are measured within radii of $0.1 R_{\mathrm{vir}}$ of the primary dark matter haloes hosting a given galaxy, excluding satellites. SFRs are averaged over the past $20 \mathrm{Myr}$ and converted into SFCs via equation (1) with $m_{\mathrm{SFC}}=m_{\mathrm{b}}$ and $t_{\mathrm{av}}=20 \mathrm{Myr}$.

The data sets contain galaxies of a range of stellar masses to increase the sample size. To combine the SFR distribution of galaxies with different stellar masses, I convert SFRs into SFCs and perform generalized linear regressions of the SFCs as a function of $\ln M_{\text {star }}$ with a $\log$ link function. ${ }^{8}$ I thus simultaneously fit for the position of the star-forming sequence as a function of stellar mass and constrain the parameters for the assumed distribution of SFCs at fixed stellar mass.

The Akaike information criterion (AIC; Akaike 1974) measures how well, relative to each other, different statistical models describe a given data set. Among the SFC distributions I tested (negative binomial, geometric, lognormal, Poisson distributions as well as their zero-inflated versions), zNBDs performed best under the AIC metric. Fig. 2 clarifies why zNBDs work so well. The lg sSFR distribution is highly asymmetric with a tail towards low values. Furthermore, there is a significant fraction of galaxies with vanishing SFRs or SFRs below the detection limit. These properties are captured by zNBDs but not by, for example, lognormal distributions.

I tested the sensitivity of the fitted model parameters (overdispersion parameter $\theta$, stellar mass scalings of the average SFR and of the excess probability $\pi$ ) by analysing additional MassiveFIRE-based samples for different redshifts and mass resolutions. The main findings are as follows: The slope and normalization of the star-forming sequence show significant changes with redshift (as expected) and only slight changes with particle resolution. The excess probability $\pi$ is not affected by redshift or mass resolution. The overdispersion $\theta$ does not depend on redshift but varies mildly with mass resolution in a not obviously systematic way.

Finally, I also explored the effect of varying the time interval over which SFRs are averaged. As expected, reducing $t_{\mathrm{av}}$ results in a smaller number of SFCs and a larger fraction of galaxies with zero SFRs. Specifically, $\pi$ increases from 20 per cent to 52 per cent as $t_{\text {av }}$ is reduced from 100 Myr to 5 Myr. Moreover, $\theta$ increases, i.e., the scatter of the star-forming sequence decreases, with increasing averaging time. However, the change in $\theta$ is relatively modest (a factor of 1.9 when $t_{\mathrm{av}}$ increases from 20 to $100 \mathrm{Myr}$ ). Furthermore, $\theta$ remains unchanged if $t_{\mathrm{av}}$ is lowered to $5 \mathrm{Myr}$. This suggests that the star formation activity of MassiveFIRE galaxies is strongly correlated on $\lesssim 20$ Myr time-scales.

\section{DISCUSSION AND CONCLUSIONS}

(z)NBDs predict significant numbers of galaxies with vanishing SFRs. As discussed in Section 2, these 'dead' galaxies form a separate component upon log-transforming SFRs, while the remaining galaxies have a unimodal log SFR distribution with an extensive

\footnotetext{
${ }^{8}$ Statistical analyses are carried out with $\mathrm{R}$ (www.r-project.org) using the standard glm function to fit Poisson and geometric distributions, the $\mathrm{glm} . \mathrm{nb}$ function from the MASs package to fit NBDs and the zeroinfl function from the PSCL package to fit zNBDs. An example regression script is provided at www.ics.uzh.ch/ feldmann/resources.html.
}

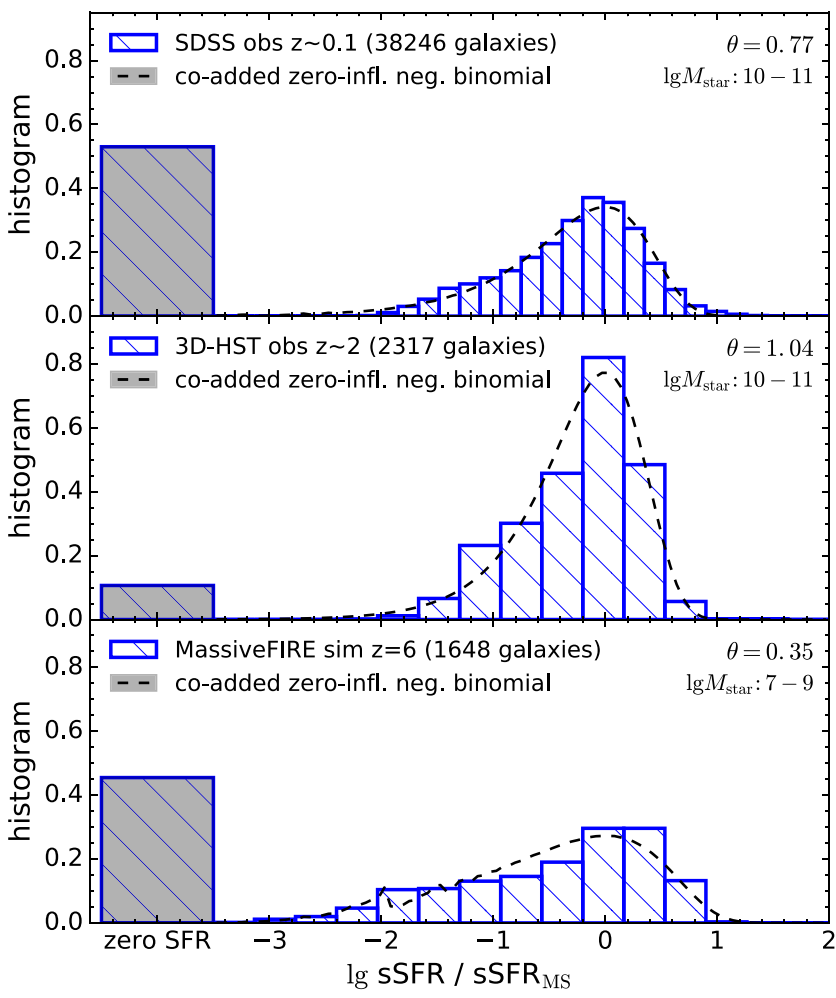

Figure 2. Distribution of sSFRs in observations and simulations. Each panel shows a histogram of the sSFR relative to peak of the star-forming sequence for mass- and redshift-selected samples of galaxies from SDSS (Chang et al. 2015), 3D-HST (Brammer et al. 2012) and MassiveFIRE (Feldmann et al. 2016). Best-fitting zNBDs (co-added relative to the $M_{\text {star-dependent peak }}$ of the star-forming sequence) are shown as dashed lines, and galaxies with undetected or zero SFRs are shown as the grey-shaded areas. The SFR distributions of the three samples are well fitted by zNBDs.

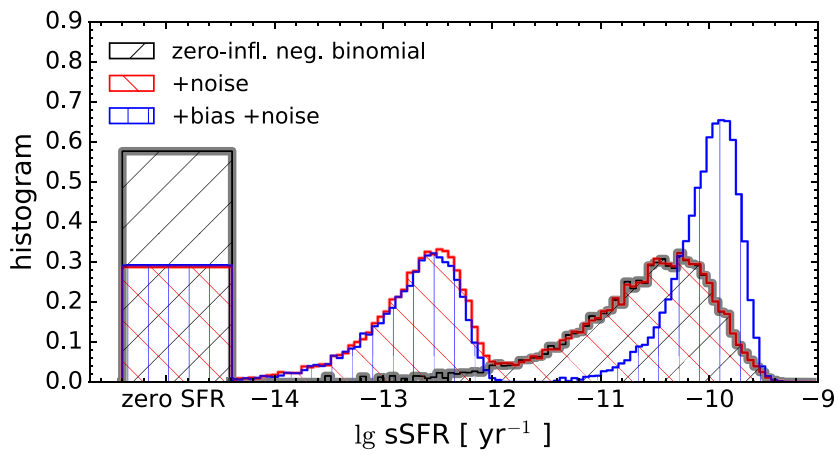

Figure 3. Difference between actual SFR distributions and inferred ones. An input SFR distribution (black histogram) is subjected to measurement uncertainties (red histogram) and non-linear biases (blue histogram). Measurement uncertainties can create the illusion of a bimodal distribution, while biases can shift and tighten the appearance of the star-forming sequence and reduce the number of galaxies with intermediate-to-low SFRs.

tail towards low SFRs. The reader may ask whether these findings are consistent with claims of an SFR bimodality (e.g. Elbaz et al. 2007; McGee et al. 2011).

I address this question in Fig. 3 in a schematic way. A detailed analysis is left for future work. Using the best fitting zNBD of the SDSS-based sample shown in the top panel of Fig. 2, I create a mock sample consisting of the SFRs of 40000 galaxies with $M_{\text {star }}=10^{10.5} \mathrm{M}_{\odot}$ (black histogram). I then subject this sample to 
non-linear biases and measurement uncertainties. ${ }^{9}$ The latter moves galaxies out of the 'dead' pile, thereby introducing in a second peak in the log SFR distribution (red histogram), while biases distort the apparent shape of the star-forming sequence (blue histogram). The combination of measurement noise and bias can create the illusion of a bimodal SFR distribution ${ }^{10}$ with low numbers of galaxies at intermediate-to-low SFRs.

Fitting the distribution of SFRs with (z)NBDs is straightforward (see footnote 8 ), and it offers substantial benefits compared with the current standard approach of fitting only the star-forming subsample with lognormals. I recommend its use for the modelling of SFR distributions both in observations [e.g. in the simplified form of (zero-inflated) gamma distributions, see footnote 7] and in simulations (where the conversion parameters $m_{\mathrm{SFC}}$ and $t_{\mathrm{av}}$ are given).

\section{ACKNOWLEDGEMENTS}

RF thanks the referee for valuable comments. RF acknowledges financial support from the Swiss National Science Foundation (grant no 157591). 3D-HST observations were taken by the 3DHST Treasury Programme (GO 12177 and 12328) under NASA contract NAS5-26555. Simulations were run under allocations SMD-14-5492, SMD-14-5189, SMD-15-5950 (NASA HEC) and AST120025, AST150045 (NSF XSEDE).

\section{REFERENCES}

Akaike H., 1974, IEEE Trans. Automat. Contr., 19, 716

Alner G. et al., 1985, Phys. Lett. B, 160, 193

Baldry I. K., Glazebrook K., Brinkmann J., Ivezić Ž., Lupton R. H., Nichol R. C., Szalay A. S., 2004, ApJ, 600, 681

Balogh M. et al., 2004, MNRAS, 348, 1355

Boswell M. T., Patil G. P., 1970, Random Counts in Models and Structures. Pennsylvania State Univ. Press, University Park, PA

Bouché N. et al., 2010, ApJ, 718, 1001

Brammer G. B. et al., 2009, ApJ, 706, L173

Brammer G. B. et al., 2012, ApJS, 200, 13

Brinchmann J., Charlot S., White S. D. M., Tremonti C., Kauffmann G., Heckman T., Brinkmann J., 2004, MNRAS, 351, 1151

Cameron A. C., Trivedi P., 2013, Book Cover. Cambridge Univ. Press, Cambridge

Carruthers P., Duong-Van M., 1983, Phys. Lett. B, 131, 116

Chang Y.-Y., van der Wel A., da Cunha E., Rix H.-W., 2015, ApJS, 219, 8

Dave R., Thompson R., Hopkins P. F., 2016, MNRAS, 462, 3265

De Souza R. S., Hilbe J. M., Buelens B., Riggs J. D., Cameron E., Ishida E. E. O., Chies-Santos A. L., Killedar M., 2015, MNRAS, 453, 1928

Dominguez A., Siana B., Brooks A. M., Christensen C. R., Bruzual G., Stark D. P., Alavi A., 2015, MNRAS, 451, 839

Dressler A. et al., 2016, ApJ, 833, 251

Dutton A. A., van den Bosch F. C., Dekel A., 2010, MNRAS, 405, 1690

Elbaz D. et al., 2007, A\&A, 468, 33

Feldmann R., Mayer L., 2015, MNRAS, 446, 1939

Feldmann R., Hopkins P. F., Quataert E., Faucher-Giguère C.-A., Kereš D., 2016, MNRAS, 458, L14

\footnotetext{
${ }^{9}$ For the bias, I assume that sSFR below sSFR $0=3 \times 10^{-10} \mathrm{yr}^{-1}$ are overestimated: $\mathrm{sSFR}_{\mathrm{obs}}=\mathrm{sSFR}_{0}\left(\mathrm{sSFR} / \mathrm{sSFR}_{0}\right)^{0.463}$, following Utomo et al. (2014). To mimic measurement uncertainties, each SFR is replaced by $\max (0$, SFR $+X)$, where $X$ is a normally distributed random variable with zero mean and standard deviation of $0.01 \mathrm{M}_{\odot} \mathrm{yr}^{-1}$.

${ }^{10}$ Similarly, colour bimodalities can arise if sSFRs are non-linearly mapped to colours, for example, if colour $=\tanh \left(3 \times \mathrm{sSFR} / \mathrm{sSFR}_{\mathrm{MS}}-1\right)$.
}

Feldmann R., Quataert E., Hopkins P. F., Faucher-Giguère C.-A., Kereš D., 2017, MNRAS, preprint (arXiv:1610.02411)

Forbes J. C., Krumholz M. R., Burkert A., Dekel A., 2014, MNRAS, 443, 168

Fumagalli M. et al., 2014, ApJ, 796, 35

Gaztanaga E., 1992, ApJ, 398, L17

Genzel R. et al., 2010, MNRAS, 407, 2091

Gladders M. D., Oemler A., Dressler A., Poggianti B., Vulcani B., Abramson L., 2013, ApJ, 770, 64

Gunn J. E., Gott J. R. I., 1972, ApJ, 176, 1

Hopkins P. F., Kere D., Onorbe J., Faucher-Giguere C.-A., Quataert E., Murray N., Bullock J. S., 2014, MNRAS, 445, 581

Kelson D. D., 2014, Astrophys. J., preprint (arXiv:1406.5191)

Kennicutt R. C., 1998, ARA\&A, 36, 189

King A., Pounds K., 2015, ARA\&A, 53, 115

Kruijssen J. M. D., 2012, MNRAS, 426, 3008

Lada C. J., Lada E. A., 2003, ARA\&A, p. 73

Lambert D., 1992, Technometrics, 34, 1

McGee S. L., Balogh M. L., Wilman D. J., Bower R. G., Mulchaey J. S., Parker L. C., Oemler A., 2011, MNRAS, 413, 996

Magdis G. E. et al., 2012, ApJ, 760, 6

Man A. W. S. et al., 2016, ApJ, 820, 11

Mancini C., Renzini A., Daddi E., Rodighiero G., Berta S., Grogin N., Kocevski D., Koekemoer A., 2015, MNRAS, 450, 763

Moustakas J. et al., 2013, ApJ, 767, 50

Mullahy J., 1986, J. Econ., 33, 341

Noeske K. G. et al., 2007, ApJ, 660, L43

Portegies Zwart S. F., McMillan S. L., Gieles M., 2010, ARA\&A, 48, 431

Rodríguez-Puebla A., Primack J. R., Behroozi P., Faber S. M., 2016, MNRAS, 455, 2592

Saintonge A. et al., 2012, ApJ, 758, 73

Salim S. et al., 2009, ApJ, 700, 161

Salmon B. et al., 2015, ApJ, 799, 183

Sánchez Almeida J., Elmegreen B. G., Muñoz-Tuñón C., Elmegreen D. M., 2014, A\&AR, 22, 71

Sargent M. T., Béthermin M., Daddi E., Elbaz D., 2012, ApJ, 747, L31

Schaye J. et al., 2015, MNRAS, 446, 521

Schreiber C. et al., 2015, A\&A, 575, A74

Scoville N. et al., 2016, ApJ, 820, 83

Shivaei I. et al., 2015, ApJ, 815, 98

Skelton R. E. et al., 2014, ApJS, 214, 24

Somerville R. S., Davé R., 2015, ARA\&A, 53, 51

Sparre M. et al., 2015, MNRAS, 447, 3548

Sparre M., Hayward C. C., Feldmann R., Faucher-Giguère C.-A., Muratov A. L., Kereš D., Hopkins P. F., 2017, MNRAS, 466, 88

Spitler L. R. et al., 2014, ApJ, 787, L36

Straatman C. M. S. et al., 2016, ApJ, 830, 51

Swinbank A. M. et al., 2010, Nature, 464, 733

Tacchella S., Dekel A., Carollo C. M., Ceverino D., DeGraf C., Lapiner S., Mandelker N., Primack Joel R., 2016, MNRAS, 457, 2790

Taylor E. N. et al., 2015, MNRAS, 446, 2144

Utomo D., Kriek M., Labbé I., Conroy C., Fumagalli M., 2014, ApJ, 783, L30

Whitaker K. E., van Dokkum P. G., Brammer G., Franx M., 2012, ApJ, 754, L29

Whitaker K. E. et al., 2014, ApJ, 795, 104

Wiel S. V., Burke-Spolaor S., Lawrence E., Law C. J., Bower G. C., 2016, preprint (arXiv:1612.00896)

Wuyts S. et al., 2007, ApJ, 655, 51

Wuyts S. et al., 2011, ApJ, 738, 106

Zhang Q., Fall S. M., 1999, ApJ, 527, L81

This paper has been typeset from a $\mathrm{T}_{\mathrm{E}} \mathrm{X} / \mathrm{L} \mathrm{T}_{\mathrm{E}} \mathrm{X}$ file prepared by the author. 